\title{
Optical Properties of a Monoclinic Insulator $\mathrm{Cu}\left(\mathrm{H}_{2} \mathrm{O}\right)_{2}(\mathrm{en}) \mathrm{SO}_{4}$
}

\author{
R. SÝkora ${ }^{a, *}$, K. Postava ${ }^{a}$, D. Legut $^{a}$ And R. TARAsenko ${ }^{b}$ \\ ${ }^{a}$ Nanotechnology Centre, Technical University of Ostrava, Ostrava, Czech Republic \\ ${ }^{b}$ Centre of Low Temperature Physics of SAS and P. J. Safarik University, Institute of Physics, Faculty of Science, \\ P. J. Safarik University, Košice, Slovakia
}

\begin{abstract}
We compare theoretically predicted, by means of $a b$-initio calculations, dielectric tensor and related experimental values obtained from Mueller-ellipsometry measurements of an insulating monoclinic (optically biaxial) crystal of $\mathrm{Cu}\left(\mathrm{H}_{2} \mathrm{O}\right)_{2}(\mathrm{en}) \mathrm{SO}_{4}$, en $=\mathrm{C}_{2} \mathrm{H}_{8} \mathrm{~N}_{2}$. We concentrate on the static limit, $\omega \rightarrow 0$.
\end{abstract}

DOI: $10.12693 /$ APhysPolA.127.469

PACS: 78.20.-e, 78.40.-q

\section{Introduction}

$\mathrm{Cu}\left(\mathrm{H}_{2} \mathrm{O}\right)_{2}(\mathrm{en}) \mathrm{SO}_{4}$ was recently investigated especially for its magnetic properties: it is an insulating (with a calculated gap of about $2 \mathrm{eV}$ ) magnetically quasi-onedimensional spin $S=1 / 2$ antiferromagnet (with the Néel temperature of $0.9 \mathrm{~K}$ ) [1-3]. In this paper we utilize the theoretical electronic structure obtained in [3] to calculate the macroscopic dielectric tensor and compare it to what can be inferred from Mueller-ellipsometry measurements (of the $\mathrm{PC}_{\mathrm{R}} \mathrm{SC}_{\mathrm{R}} \mathrm{A}$ type) carried out in the 190 to $1700 \mathrm{~nm}$ range $(6.5-0.7 \mathrm{eV})$. The material crystal belongs to the monoclinic system and thus is optically biaxial, i.e. displays anisotropy. Contrary to orthorhombic crystals (biaxial crystals of highest crystallographic symmetry), the crystallographic axes of a monoclinic crystal do not fix all the axes of the dielectric frame, defined as the frame where the real part of the (generally complex) dielectric tensor is diagonal. This makes the situation complex on the one hand, but on the other hand more experimental features, such as the orientation of the dielectric frame, can be checked against theory.

\section{Geometry}

The material belongs to the prismatic class of the monoclinic crystal system, i.e., has the 2 /m point-group symmetry [4]. As is conventionally done, we call the symmetry axis the $b$ axis. This crystallographic axis is perpendicular to the other two, $a$ and $c$, axes, and the latter form an angle of $105.5^{\circ}$, see Fig. 1 . The ellipsometry measured light reflection from a crystal facet parallel to the $a c$ plane (with the $b$ axis perpendicular to it). The orientation (Miller indices) of the facet, the shaded area in Fig. 1, was checked by X-ray diffraction. Generally, frequencydependent (macroscopic) dielectric tensor $\varepsilon(\omega)$ expressed

* corresponding author; e-mail: rudolf.sykora@vsb.cz
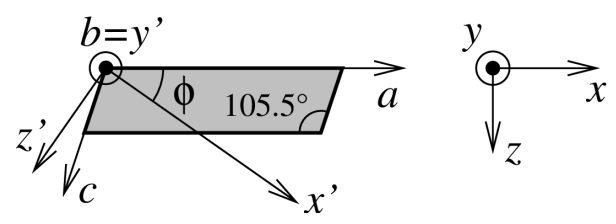

Fig. 1. The relation between the crystallographic axes $a, b, c$, the considered crystal facet (shaded area) parallel to the $a c$ plane, and used orthogonal (righthanded) systems $(x y z)$ and $\left(x^{\prime} y^{\prime} z^{\prime}\right)$. The $b, y$ and $y^{\prime}$ axes coincide, the $(x y z)$ and $\left(x^{\prime} y^{\prime} z^{\prime}\right)$ systems are mutually rotated by the angle $\phi$ around the $y$ axis. The $\left(x^{\prime} y^{\prime} z^{\prime}\right)$ system is the principal frame for $\Re \varepsilon$. We define (positive) $x^{\prime}$ to lie in the quadrant of positive $x$ and $z$ axes, and $\phi \in\langle 0,90\rangle^{\circ}$ there.

in any orthogonal coordinate system $(x y z)$ is a complex symmetric matrix, $\varepsilon_{i j}(\omega)=\varepsilon_{j i}(\omega) ; i, j \in\{x, y, z\}$. Hence its real and imaginary part can be individually diagonalized by an ( $\omega$-dependent) orthogonal transformation, which, however, may be different for each part; we call the resulting frames the principal frame for the real and imaginary part, or alternatively the dielectric and absorption frame, respectively. Fortunately, the monoclinic symmetry requires that the $b$ axis be also one of the principal-frame axes (for both parts) [5]. This implies (i) that $\varepsilon$ expressed in the $(x y z)$ system whose orientation is given in Fig. 1 must have the form $\left(\begin{array}{ccc}\varepsilon_{x x} & 0 & \varepsilon_{x z} \\ 0 & \varepsilon_{y y} & 0 \\ \varepsilon_{z x} & 0 & \varepsilon_{z z}\end{array}\right)$ and (ii) the existence of an orthogonal $\left(x^{\prime} y^{\prime} z^{\prime}\right)$ coordinate system whose $y^{\prime}$ axis coincides with the crystal $b$ axis (and the $y$ axis) and in which the real part of $\varepsilon$ is diagonal; there $\Re \varepsilon=: \operatorname{diag}\left(\varepsilon_{x^{\prime}}, \varepsilon_{y^{\prime}}, \varepsilon_{z^{\prime}}\right)$. The relation between $(x y z)$ and $\left(x^{\prime} y^{\prime} z^{\prime}\right)$ is a simple rotation about $y$ by the angle $\phi$. (Similarly there is a rotation about $y$ which diagonalizes the imaginary part of $\varepsilon$, but this will be of no interest to us now.)

When light of a frequency at which the material is nonabsorbing $(\Im \varepsilon=0)$ is shed on the sample and its ray lies in either the $x^{\prime} b$ or the $z^{\prime} b$ plane, then due to the above-discussed diagonality of $\Re \varepsilon$ there is no conversion 
between the $s$ and $p$ polarizations (i.e., the Jones matrix is of the $\left(\begin{array}{cc}r_{p p} & 0 \\ 0 & r\end{array}\right)$ form, with vanishing $r_{s p}$ and $r_{p s}$; we ignore any possible depolarization, which is supported by the experiment). This is true for any angle of incidence (in the ideal-geometry setup); only the azimuth, $\phi$, plays a role. In the following we primarily want to compare a theoretical prediction of $\phi$ to the experiment in the limit $\omega \rightarrow 0$. In this limit (i) the absorption is low (the material has a gap), (ii) $\varepsilon$ dispersion is weak and we can then expect that $\phi$ only weakly depends on frequency, (iii) we may trust $a b$-initio calculations the most.

\section{Computational methodology}

Both electronic-structure and optics calculations were performed using the VASP code [6] within the density functional theory, using the projector-augmented-wave pseudopotential method with the generalized gradient approximation parameterized by Perdew, Burke, and Ernzerhof in its GGA + U extension as implemented in the code following Dudarev [7] and using $U=5.5 \mathrm{eV}$. This value follows from the analysis in [3]. The material conventional unit cell contains four chemical formula units $(Z=4), 96$ atoms altogether. The Brillouin zone was sampled on a $k$-point mesh with $6 \times 4 \times 5$ points. Used lattice parameters and atomic positions are reported in [2]. We calculated both the frequency-dependent $\varepsilon$ (using the LOPTICS method of VASP with NBANDS=800), and the static limit (using the LEPSILON method) and checked these two agree. By means of diagonalizing the calculated $\varepsilon$ the theoretic prediction for $\phi$ is obtained.

\section{Results}

Figures 2 and 3 show the calculated real and imaginary parts of the dielectric tensor expressed in the ( $x y z)$ frame. The anisotropic behaviour is clearly visible in both parts. Looking at the real part and considering the static limit we see that the off-diagonal element $\varepsilon_{z x}$ is much smaller then the diagonal elements, however, is comparable to the diagonal elements difference. This has two consequences: (i) Diagonalization of $\varepsilon$ will yield eigenvalues close to what can be read from the inset (actually, as should be clear, the $\Re \varepsilon_{y y}$ directly gives $\varepsilon_{y^{\prime}}$, while only the $x$ and $z$ components mix). Mathematically this follows from the fact that diagonalization of the matrix $\left(\begin{array}{c}\bar{\varepsilon}+\Delta \\ \delta \\ \bar{\varepsilon}-\Delta\end{array}\right)$ yields eigenvalues $\varepsilon=\bar{\varepsilon} \pm \sqrt{\Delta^{2}+\delta^{2}}$. (ii) The azimuth angle of the dielectric frame need not be small (as one could wrongly expect at first). The essence of this fact can be understood from diagonalization of the matrix $\left(\begin{array}{ll}1 & \delta \\ \delta & 1\end{array}\right)$, which always leads to a $45^{\circ}$ rotation irrespective of the value of $\delta$ (but for the strict $\delta=0$ case).

The calculated elements of the diagonalized $\Re \varepsilon$ at $\omega=$ 0 are $\varepsilon_{x^{\prime}}=2.55, \varepsilon_{y^{\prime}}=2.83, \varepsilon_{z^{\prime}}=2.74$. The calculated azimuth $\phi$ which diagonalizes $\varepsilon$ is given in Fig. 4 for the whole measured spectrum.

Turning attention to the spectrum of $\Im \varepsilon$, Fig. 3, we see no absorption till about $2 \mathrm{eV}$ and then a marked absorption in the blue region. This is not in accord with reality

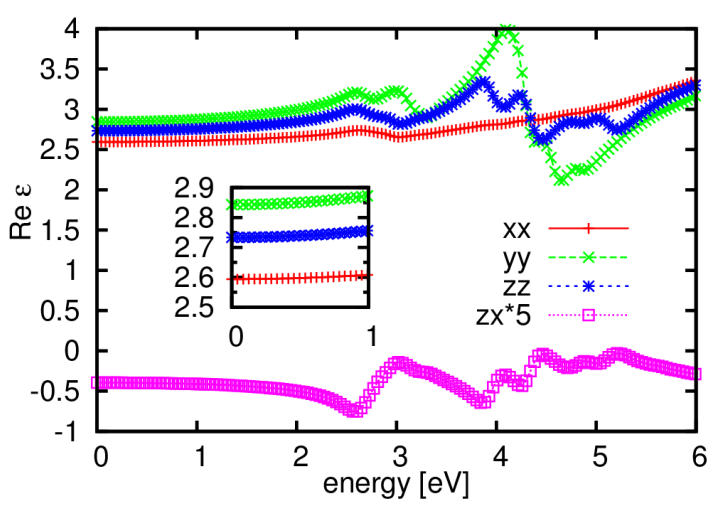

Fig. 2. Calculated elements of $\Re \varepsilon$ in the (xyz) frame; elements not shown are zero (but $\varepsilon_{x z}=\varepsilon_{z x}$ ). In the inset the $\omega \rightarrow 0$ limit of the diagonal elements is detailed.

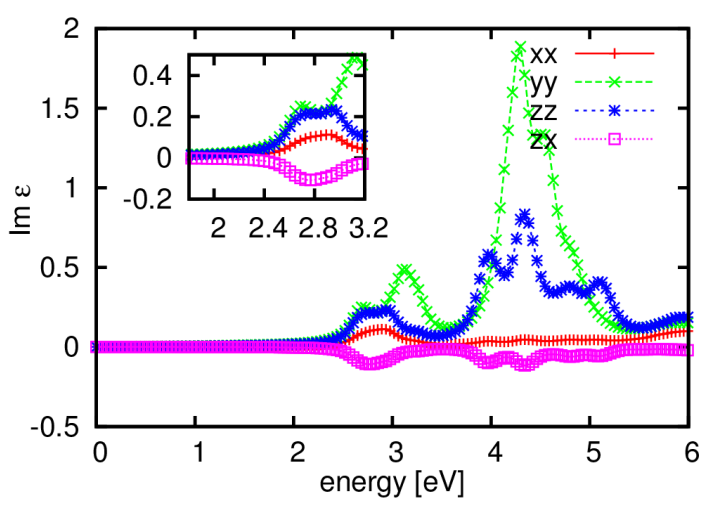

Fig. 3. Calculated elements of $\Im \varepsilon$ in the ( $x y z)$ frame; elements not shown are zero (but $\varepsilon_{x z}=\varepsilon_{z x}$ ). The inset depicts the visible part of the spectrum.

since the crystal is apparently blue, hence we would expect absorption in the yellow region and then much lower or no absorption in the blue region. This discrepancy is one of the reasons why we concentrate mainly on the static limit in this contribution.

In Fig. 4 the calculated azimuthal rotation $\phi$ needed to diagonalize the real part of the dielectric tensor is displayed. As expected, the angle shows a smooth behaviour

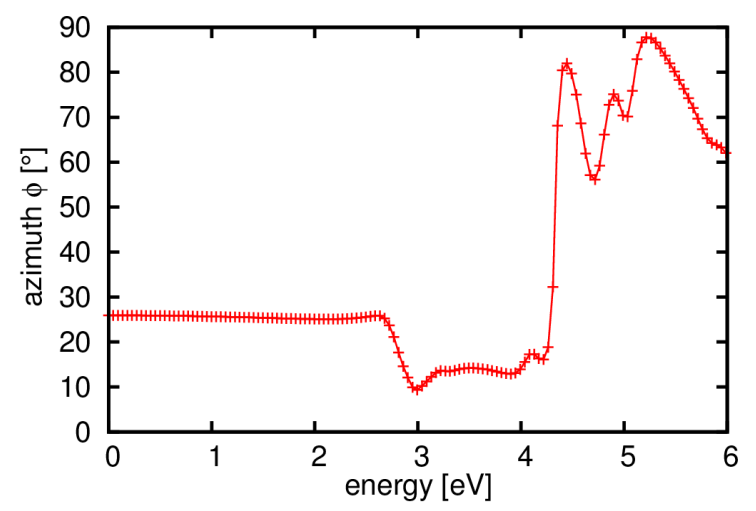

Fig. 4. Calculated azimuth, $\phi$, of the dielectric frame as a function of light energy. 
towards the static limit with a nearly constant value of about $25^{\circ}$. At this azimuth as well as related azimuths differing by multiples of $90^{\circ}$ we should see no polarization conversion. This is almost seen in the experiment, Fig. 5, at the angles of (approximatively) 65, 155, 245 and 335 degrees (these are complements to $360^{\circ}$ of the angles just mentioned; the beam azimuth rotates effectively in the opposite sense than the sample). Almost, first because we see the curves do not always drop completely to zero. This, however, may well be just an artefact of not having a fine enough azimuthal step. Second, the minima do not really coincide. Our current opinion on this is that the sample alignment was not perfect inasmuch the $b$ axis was not identical with the axis of azimuthal rotation. (The best we can then do to predict the minima positions for the ideal alignment is to take the averages; these are the above numbers.) The misalignment may further also be the reason for why the sample does not look the same after a rotation by $180^{\circ}$ (which it would strictly have to if the $b$ axis were the axis of rotation).

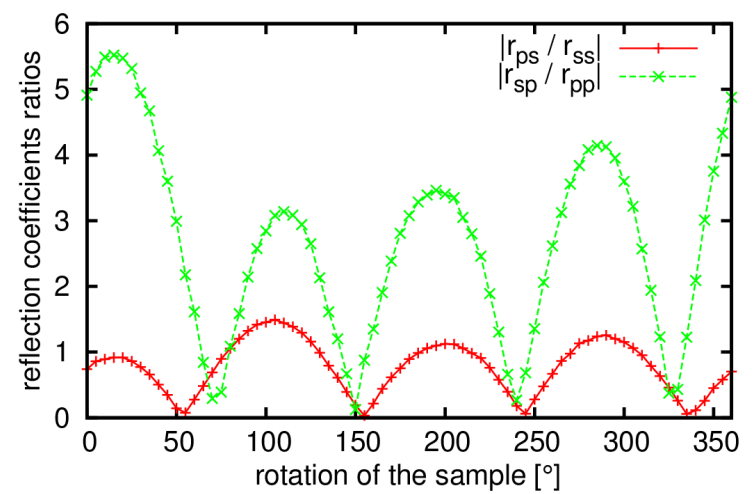

Fig. 5. Measured ratios $\left|r_{p s} / r_{s s}\right|$ and $\left|r_{s p} / r_{p p}\right|$ as a function of rotation of the sample for light of $1240 \mathrm{~nm}$ and $45^{\circ}$ incidence angle. We define the Jones matrix as $\left(\begin{array}{cc}r_{p p} & r_{p s} \\ r_{s p} & r_{s s}\end{array}\right)$

\section{Conclusions}

We have calculated the complex frequency-dependent macroscopic dielectric tensor for the material. The nonzero-frequency results are not satisfactory: the predicted colour is wrong. In this respect we note that we have only used the ground-state DFT framework, which although suitable for the static limit is not really the right tool for determining non-zero frequency behaviour. (The so-called GW approximation together with the BetheSalpeter equation, or alternatively the TDDFT framework, should be used instead. In this way also excitonic effects, which may exist and modify the spectrum, could be included.)

On the other hand, the theoretically predicted orientation of the dielectric frame in the static limithere the most studied aspect, related to the system's monoclinicity - agrees with the experiment well.
In the static limit we have predicted the three principal indices of refraction. It would be desirable to compare these to the experiment. Unfortunately, at this point we seem to need some more information from the experiment that would help us disentangle the action of the individual refraction-index components. (For instance, in the 'diagonalized' setup the reflection of $s$ polarization depends only on either $n_{x^{\prime}}$, or $n_{z^{\prime}}$. From the knowledge of incidence angle and the absolute value of $\left|r_{s s}\right|$ at least these two refractive indices could be calculated. Such an absolute value is not readily available, though.)

\section{Acknowledgments}

Our thanks belong to the experimental group of A. Orendáčová at the UPJŠ in Košice, which prepared and provided the sample as well as checked the crystal facet orientation with X-ray diffraction. The paper was elaborated in the framework of the project New creative teams in priorities of scientific research, reg. no. CZ.1.07/2.3.00/30.0055, supported by Operational Programme Education for Competitiveness and co-financed by the European Social Fund and the state budget of the Czech Republic. D. Legut was supported by the Grant Agency of the Czech Republic under reg. no. 1330397S and by OpVaVpl project CZ.1.07/2.3.00/20.0074. R. Tarasenko was supported by the project VEGA 1/0143/13 of the Scientific Grant Agency of the Ministry of Education of Slovak Republic and the Slovak Academy of Sciences. This research used resources of the National Supercomputing Center IT4Innovations (LM2011033), supported by the Centre of Excellence project number CZ.1.05/1.1.00/02.0070. This work acknowledges COST Action MP 1306 EUSpec.

\section{References}

[1] M. Kajňaková, M. Orendáč, A. Orendáčová, A. Vlček, J. Černák, O.V. Kravchyna, A.G. Anders, M. Bałanda, J.-H. Park, A. Feher, M.W. Meisel Phys. Rev. B 71, 014435 (2005).

[2] R. Sýkora, D. Legut, U.D. Wdowik, Acta Phys. Pol. $A$ 126, 50 (2014).

[3] R. Sýkora, D. Legut, J. Appl. Phys. 115, 17B305 (2014).

[4] V. Manríquez, M. Campos-Vallette, N. Lara, N. González-Tejeda, O. Wittke, G. Díaz, S. Diez, R. Muñoz, L. Kriskovic, J. Chem. Cryst. 26, 15 (1996).

[5] R.P. Feynman, R.B. Leighton, M.Sands, Feynman Lectures on Physics, Ch. 31.3.

[6] G. Kresse, J. Furthmüller, Phys. Rev. B 54, 11169 (2014).

[7] S.L. Dudarev, G.A. Botton, S.Y. Savrasov, C.J. Humphreys, A.P. Sutton, Phys. Rev. B 57, 1505 (1998). 